\title{
Double magnetic reorientation transition in thin garnet films
}

\author{
L. T. Tsymbal, ${ }^{1}$ G. N. Kakazei, ${ }^{2}$ and Ya. B. Bazaliy ${ }^{3, *}$ \\ ${ }^{1}$ O. Galkin Donetsk Physics and Technology Institute, NASU, Donetsk/Kyiv, Ukraine \\ ${ }^{2}$ Institute of Physics for Advanced Materials, Nanotechnology and Photonics (IFIMUP)/Departamento de Fisica e Astronomia, \\ Universidade do Porto, Porto 4169-007, Portugal \\ ${ }^{3}$ Department of Physics and Astronomy, University of South Carolina, Columbia, South Carolina 29208, USA
}

(Received 29 July 2013; revised 23 August 2020; accepted 28 September 2020; published 2 November 2020)

\begin{abstract}
Thin garnet films with perpendicular anisotropy are enjoying renewed attention in the context of research on heavy metal/ferromagnetic insulator bilayer devices with tunable electric and magnetic properties. We show that two temperature-induced magnetic orientation transitions observed in such films have different origins. One transition occurs between the uniform, in-plane magnetized state and the stripe domain state, and is governed by the bulk material properties. The other occurs between the domain state and the perpendicular magnetized uniform state, and is governed by the physics of domain nucleation.
\end{abstract}

DOI: 10.1103/PhysRevResearch.2.043170

\section{INTRODUCTION}

Thin garnets films currently enjoy a resurgence of interest within the field of "insulator spintronics" that studies the interconversion of electronic and magnonic forms of spin currents [1-7]. Garnets, with their low magnetic damping constants, are prime materials in this field. Substantial efforts are devoted to the studies of garnet/heavy metal bilayers, where the magnetism of garnet insulators is interacting with the electron transport in conductors with strong spin orbit interactions [8-14]. While many studies use perpendicular magnetized films [15-23], recent experiments also investigated the consequences of magnetization rotations at orientation transitions [24] and magnetization vanishing at the garnet's magnetic compensation temperature $[9,21]$.

In view of the importance of the role played by the garnet film magnetic orientation transitions, a detailed investigation of their nature appears useful and desirable. In the present work, we perform an experimental investigation of the transition between perpendicular and in-plane states that is driven by the change of magnetization's absolute value with temperature. We discuss the deficiencies of the previous theoretical explanation of two sequential reorientation transitions observed in experiments and suggest a better physical picture of the phenomenon.

Thin magnetic films with perpendicular anisotropy exhibit orientation phase transitions driven by a competition between the anisotropy energy and the dipole-dipole interaction (demagnetization) energy. We will assume that the thickness

\footnotetext{
*bazaliy@mailbox.sc.edu

Published by the American Physical Society under the terms of the Creative Commons Attribution 4.0 International license. Further distribution of this work must maintain attribution to the author(s) and the published article's title, journal citation, and DOI.
}

of the film is much larger than the lattice constant and it is possible to describe it as a continuous three-dimensional magnetic material characterized by certain magnetization and anisotropy energy density. The simplest model exhibiting an orientation transition assumes a uniformly magnetized film with magnetization vector $\mathbf{M}=M \mathbf{n}$ of constant magnitude $M$ and direction given by a unit vector $\mathbf{n}$. The film is assumed to have a perpendicular crystalline anisotropy, so that in the absence of external field its energy equals

$$
E=-\frac{1}{2} K n_{z}^{2}+2 \pi M_{z}^{2}=-\frac{1}{2} K_{\mathrm{eff}} n_{z}^{2},
$$

where $z$ is the axis perpendicular to the film plane, $K>0$ is the anisotropy constant, $2 \pi M_{z}^{2}$ represents the demagnetization energy of an infinite film, and $K_{\text {eff }}=K-4 \pi M^{2}$. In this model, the magnetization points perpendicular to the plane of the film for $K_{\text {eff }}>0$ and lies in plane otherwise. A firstorder orientation transition between the two states happens at $K_{\text {eff }}=0$.

In reality, magnetization $\mathbf{M}(\mathbf{r})$ may be spatially nonuniform and vary both in-plane and along $z$, forming magnetic domains. The anisotropy constant can be space dependent as well, e.g., when it is produced by the surface anisotropy that acts only at the top and bottom interfaces of the film. In this case, the energy has to be expressed in an integral form

$$
\begin{aligned}
E= & \int d x d y \int_{0}^{d} d z\left[-\frac{K(\mathbf{r})}{2} n_{z}^{2}(\mathbf{r})+\frac{A}{2}\left(\frac{\partial n_{i}}{\partial r_{k}}\right)^{2}\right. \\
& -\mathbf{H} \cdot \mathbf{M}(\mathbf{r})]+E_{\operatorname{dip}}\{\mathbf{M}(\mathbf{r})\}
\end{aligned}
$$

where $d$ is the film thickness, $A$ is the spin stiffness, $\mathbf{H}$ is the applied magnetic field, and $E_{\text {dip }}$ is the dipole-dipole interaction energy

$$
E_{\mathrm{dip}}=\iint \frac{\operatorname{div} \mathbf{M}(\mathbf{r}) \operatorname{div} \mathbf{M}\left(\mathbf{r}^{\prime}\right)}{\left|\mathbf{r}-\mathbf{r}^{\prime}\right|} d^{3} r d^{3} r^{\prime}
$$




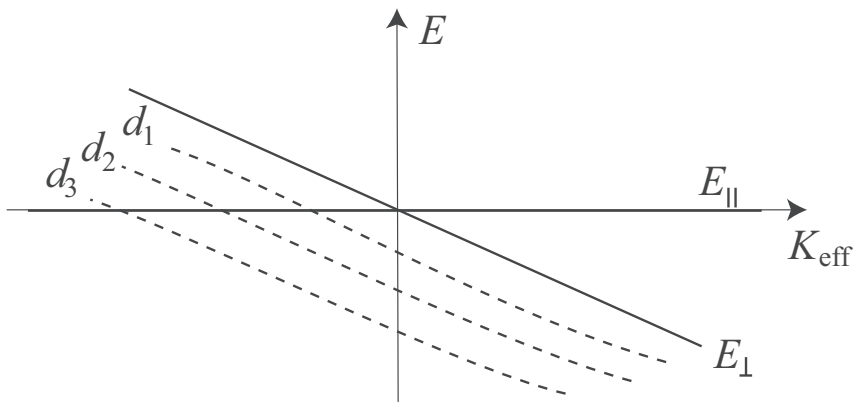

FIG. 1. Qualitative sketch of the energies of various magnetic states of a film with perpendicular magnetic anisotropy. Solid lines give the energies of the uniform states: $E_{\|}=0$ for $\mathbf{M}$ in-plane, $E_{\perp}=-K_{\text {eff }} / 2$ for M perpendicular to the film's plane. Dashed lines give the energies of stripe-domain states for several film thicknesses $d_{1}<d_{2}<d_{3}$.

with integrations performed over the volume of the film. The quantity - divM is known as the "magnetic charge" and includes the "surface magnetic charge" term $(\mathbf{M} \cdot \boldsymbol{v}) \delta_{S}$, where $\boldsymbol{v}$ is the outward normal and $\delta_{S}$ is the $\delta$ function on the film surface. In the case of a uniform magnetization, all demagnetization energy is due to the latter term. With spatial dependencies being allowed, the problem acquires two additional parameters: spin stiffness $A$ and film thickness $d$.

When magnetic structures with spatially varying $\mathbf{M}(\mathbf{r})$ are taken into account, orientation transitions are no longer restricted to those between uniform states. Transitions between uniform and nonuniform states are also possible and well documented. One example is the transition induced by the change of $d$ : The film has a stripe domain configuration above the critical thickness, $d>d_{c}$, and is magnetized in-plane for $d<d_{c}$ [25-32]. Figure 1 sketches the energy of the film as a function of $K_{\text {eff }}$ for uniform and nonuniform states. The latter are assumed to be stripe-domain structures with alternating up- and down-magnetized domains. At $K_{\text {eff }}<0$, the increase of $d$ makes the energy of a stripe-domain state lower than that of a uniform in-plane state. At $d \gg d_{c}$, one observes a "strong" stripe phase, where the domain sizes are much larger than the widths of the walls separating them, so that both the domains and the walls between them are well defined. At $d$ just a little larger than $d_{c}$, the film may go into a "weak" stripe phase, where the wall width becomes comparable to the domain size. In that regime, there is no clear separation between the domains and the walls, and the magnetization smoothly meanders inside the film.

Alternatively, an orientation transition can be observed in a film of constant thickness if parameters $K, M$, or $A$ are temperature dependent. In this case, transition will be induced by a temperature change. Such transition was thoroughly investigated in ultrathin films with perpendicular anisotropy [33], where $K_{\text {eff }}(T)$ is positive at low temperatures and changes sign at high temperatures [34]. Then, according to Fig. 1, the domain structure state is the energy minimum at low temperatures, while the in-plane uniform state is realized at high temperatures [35-37]. The nature of the transition between the two states (first or second order) depends on the system parameters and was a subject of many studies [37-41].
In the present paper, we study the relatively thick films with substantial temperature dependence of magnetization $M(T)$. A bismuth substituted thulium iron garnet film was used in our experiments. We have been motivated by earlier work on films with the same nominal composition $[42,43]$, where it was concluded that the temperature-driven reorientation proceeds in a series of two second-order phase transitions at temperatures $T_{1}$ and $T_{2}\left(T_{2}>T_{1}\right)$. The film was found to be magnetized in plane at $T<T_{1}$ and perpendicular to plane for $T_{2}<T<T_{c}$. References [42,43] offered an explanation for this sequence of transitions based on the known temperature dependence of magnetization. They assumed spatially uniform $\mathbf{M}$ at all temperatures and added a quartic term to the perpendicular anisotropy energy expression (1)

$$
E=-\frac{K_{\mathrm{eff}}(T)}{2} n_{z}^{2}+\frac{K_{2}}{4} n_{z}^{4} .
$$

Temperature dependence of magnetization $M(T)$ was assumed to be the sole reason for the $K_{\text {eff }}(T)$ dependence. Magnetization is large at low temperatures, leading to $K_{\text {eff }}(0)<0$. As the temperature is raised, $M(T)$ decreases continuously until reaching zero at the magnetic compensation point $T_{c}>T_{2}$. Accordingly, $K_{\text {eff }}(T)$ increases from a negative $K_{\text {eff }}(0)$ to a positive $K_{\text {eff }}\left(T_{c}\right)=K>0$, i.e., evolves in the opposite direction as compared to the case of ultrathin films. It is well known from the classic studies of orthoferrites $[44,45]$ that a quartic term in the energy expression (3) produces the required sequence of two second-order transitions with $T_{1}$ given by the condition $K_{\text {eff }}\left(T_{1}\right)=0$ and $T_{2}$ by $K_{\text {eff }}\left(T_{2}\right)=K_{2}$.

While Refs. [42,43] succeeded in giving a qualitative explanation of the reorientation transitions, some problems were still present. First, the values of $T_{1}$ and $T_{2}$ strongly depended on the type of experiment (magnetization versus ferromagnetic resonance measurements). Second, in external magnetic field $H$ the induced shift of the second transition point $T_{2}(H)$ was much larger than that of $T_{1}(H)$. Third, measurements inside the $\left[T_{1}, T_{2}\right]$ interval, where a tilted phase is predicted by minimization of the energy function (3), did not show partial magnetization either in the in-plane or in the out-of-plane directions. We have performed a new series of experiments to look into these issues and check whether experimental findings can be better explained by taking into account nonuniform magnetic states.

\section{EXPERIMENT}

Measurements were performed on the substituted thulium iron garnet film with the composition $\mathrm{Tm}_{2.14} \mathrm{Bi}_{0.8} \mathrm{~Pb}_{0.06} \mathrm{Fe}_{3.1} \mathrm{Ga}_{1.9} \mathrm{O}_{12}$. The thickness of the film was $d \approx 5 \mu \mathrm{m}$. It was grown on [111] oriented gadolinium gallium garnet by the liquid-phase epitaxy method and annealed at $1050 \mathrm{C}$ in oxygen for approximately $50 \mathrm{~min}$. The magnetic moment $\mathcal{M}$ was measured using a superconducting quantum interference device magnetometer Quantum Design MPMS-5 (temperature range 1.7-400 K, magnetic field range $0-50 \mathrm{kOe}$ ). In all measurements, the magnetometer recorded the component of $\mathcal{M}$ along the direction of the applied field. Measurements were performed with the field perpendicular 


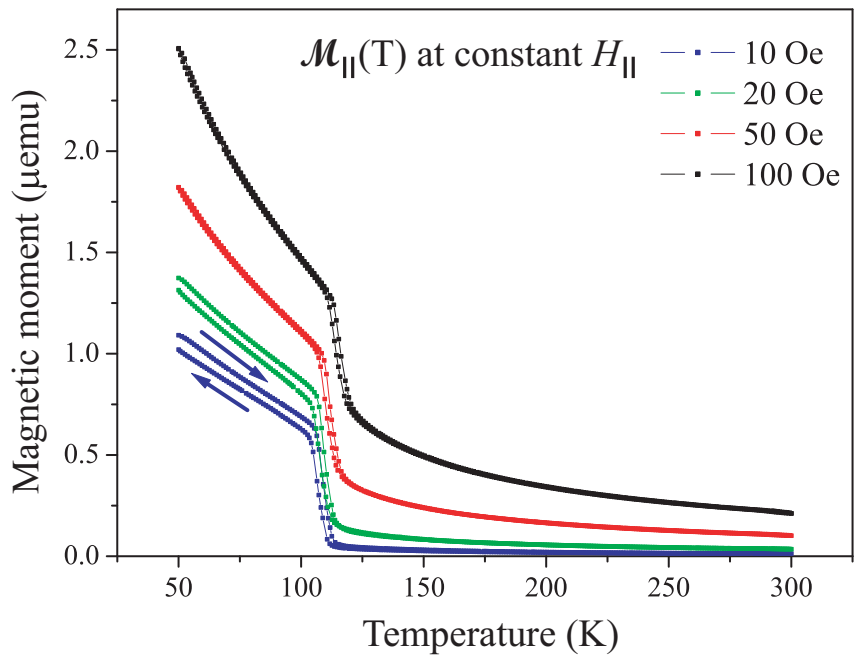

FIG. 2. In-plane $\mathcal{M}_{\|}(T)$ at different values of applied field $H_{\|}$. The transition at $T_{1} \approx 120 \mathrm{~K}$ gradually moves to higher temperatures as the field is increased.

to the film plane, $\mathbf{H} \| z$, and in-plane, $\mathbf{H} \perp z$. We use $H_{z}$ and $H_{\|}$to denote the field magnitudes in those two orientations.

First, the temperature dependencies $\mathcal{M}_{\|}(T)$ (Fig. 2) and $\mathcal{M}_{z}(T)$ (Fig. 3) were obtained. Measurements were started at room temperature and large fields $H_{||}, H_{z} \approx 1 \mathrm{kOe}$ that saturated the film magnetization in the applied field direction. Then the field was lowered to a small value, and a temperature sweep was conducted at constant $H_{\|}$or $H_{z}$.

We start our discussion from the $H_{\|}, H_{z}=0$ sweeps. In agreement with the literature data $[42,43]$, in zero external field the $\mathcal{M}(T)$ dependence shows two orientation transitions (for our film $T_{1} \approx 120 \mathrm{~K}$ and $T_{2} \approx 220 \mathrm{~K}$ ). Almost no hysteresis of either $\mathcal{M}_{\|}(T)$ or $\mathcal{M}_{z}(T)$ curves was observed near

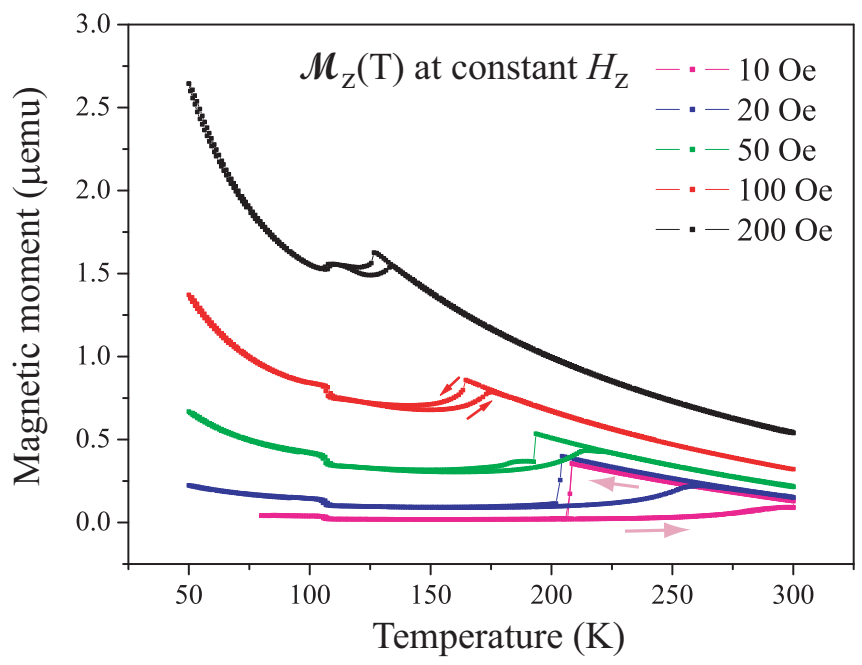

FIG. 3. Out-of-plane $\mathcal{M}_{z}(T)$ at different values of applied field $H_{z}$. Downward magnetization jump at $T_{2} \approx 220 \mathrm{~K}$ moves to lower temperatures as $H_{z}$ is increased. The curves exhibits a characteristic "triangular hysteresis" pattern above $T_{2}$. The lower transition is reflected as a small jump at $T_{1} \approx 120 \mathrm{~K}$ and subsequent increase of $\mathcal{M}_{z}$ (see the discussion section). the $T_{1}$ transition. At the $T_{2}$ transition, we found hysteresis loops of $\mathcal{M}_{z}(T)$ with characteristic "triangular" shapes in all of the applied fields $H_{z}$ (Fig. 3). Magnetic moment of the film clearly points in plane at $T<T_{1}$. At $T>T_{2}$, the moment is perpendicular to the plane on the upper branch of the triangular hysteresis loop and equals zero on the lower branch. In between the two transition temperatures, both components of the moment vanish. Except for the range between $T_{1}$ and $T_{2}$, the absolute value of $\mathcal{M}$ is found to be continuously increasing as the temperature is decreased. A compensation point was not observed, presumably because in our film it was above room temperature. The increase of magnetization produces the decrease of effective anisotropy constant $K_{\text {eff }}(T)$ with lowering temperature. This is consistent with the sign change of $K_{\text {eff }}$ from positive to negative near $T_{1}$. The energy expression (3) used in Refs. [42,43] then indeed predicts an out-of-plane phase at high temperatures and an in-plane phase at low temperatures, in agreement with the experimental results for $T<T_{1}$ and $T>T_{2}$. In the $\left[T_{1}, T_{2}\right]$ interval, Eq. (3) predicts a canted phase and nonzero values of both $\mathcal{M}_{z}$ and $\mathcal{M}_{\|}$components $[44,45]$, showing a disagreement with the experimental findings.

At finite values of $H$, the $\mathcal{M}(T)$ curves look qualitatively the same but change quantitatively. First, the transition points are shifted, so that $T_{1}=T_{1}\left(H_{\|}\right)$and $T_{2}=T_{2}\left(H_{z}\right)$. Second, the measured $\mathcal{M}_{\|}$and $\mathcal{M}_{z}$ components acquire finite values in the regions where they were previously equal to zero. Third, at finite $H_{z}$ the $\mathcal{M}_{z}(T)$ curves (Fig. 3) exhibit two jumps: a large one at the $T_{2}$ transition and a small one at the $T_{1}$ transition.

The triangular temperature hysteresis observed near $T_{2}$ looks identical to the hysteresis pattern observed in bulk single-crystal orthoferrites [46-49], where it was caused by the domain structure formation in the sample. The analogy with the case of bulk orthoferrites goes further. Near $T_{2}$, the field hysteresis loops $\mathcal{M}_{z}\left(H_{z}\right)$ exhibit one of the two characteristic shapes with jumps: A "double triangle" shape [Fig. 4(a)] or a "triangular tail" shape [Fig. 4(b)]. Similar to the case of orthoferrites $[46,47]$, one finds that the jumps on the $\mathcal{M}_{z}(T)$ and $\mathcal{M}_{z}\left(H_{z}\right)$ hysteresis curves reflect the same underlying phenomenon since both happen upon crossing the same, experimentally measurable line on the $\left(T, H_{z}\right)$ parameter plane. Moreover, the connection between the double triangle shape of the field hysteresis loops and the domain structure formation in thin magnetic films is well documented in the literature [27]. All of the above suggests that the $T_{2}$ transition may be related to the physics of magnetic domains and domain walls, rather than determined by the bulk magnetic energy (3). Proving this conjecture is the main goal of the present paper.

We have additionally performed the $\mathcal{M}_{z}(T)$ measurements in fixed negative fields $H_{z}<0$ (Fig. 5). This meant initially saturating the magnetization in the up direction by $H_{z} \approx+1$ $\mathrm{kOe}$ at room temperature and then lowering the field to a small negative value between -10 Oe and -100 Oe. Negative fields were weak enough not to switch the film from the upsaturated state. After the initial field drop, a temperature scan was performed in a fixed negative $H_{z}$. One can observe how the shapes of the $\mathcal{M}_{z}(T)$ curves continuously evolve from the $H_{z}>0$ case (Fig. 3) to the $H_{z}<0$ case (Fig. 5). Regardless 

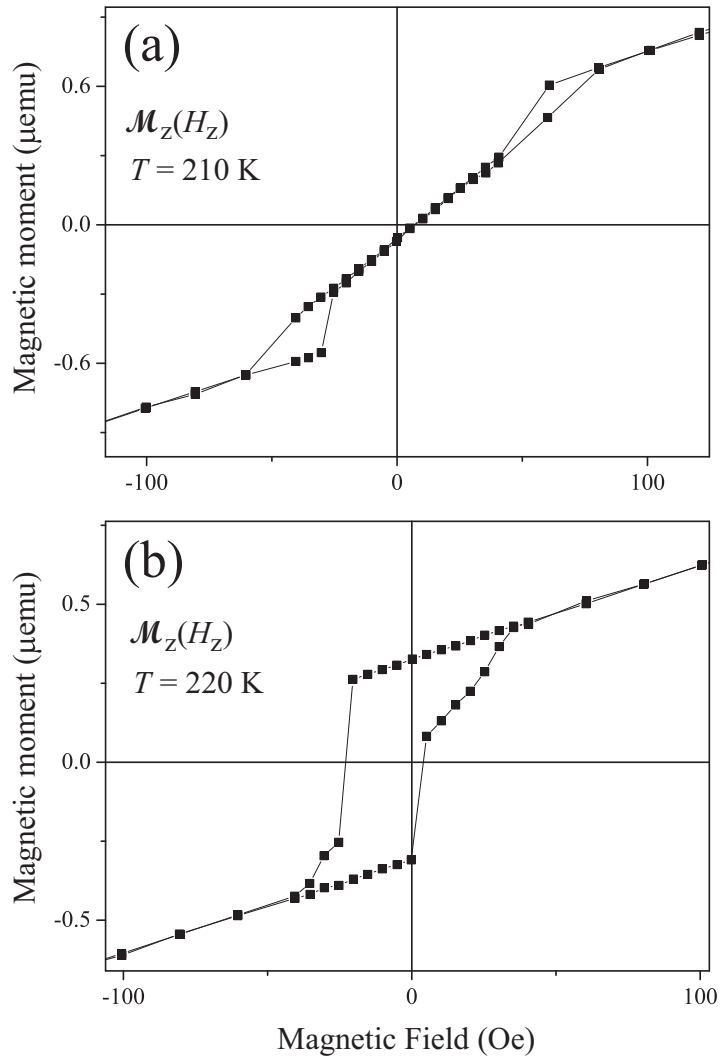

FIG. 4. Hysteresis loops $\mathcal{M}_{z}\left(H_{z}\right)$ of the film in a perpendicular magnetic field. (a) "Double-triangle" loop. (b) “Triangular tail” loop.

of the sign of $H_{z}$ they exhibit a larger jump at $T_{2}$ and a smaller one at $T_{1}$.

The field-induced shift of the $T_{2}$ transition temperature can be fitted by an approximate formula $T_{2}\left(H_{z}\right) \approx T_{2}(0)+$ $\left(d T_{2} / d H_{z}\right) H_{z}$ with $d T_{2} / d H_{z} \approx-0.5 \mathrm{~K} / \mathrm{Oe}$, which works for both positive and negative fields. The field-induced shift of the $T_{1}$ transition temperature is much smaller and has an opposite sign. From Fig. 2 we get $d T_{1} / d H_{\|} \approx 0.1 \mathrm{~K} / \mathrm{Oe}$.

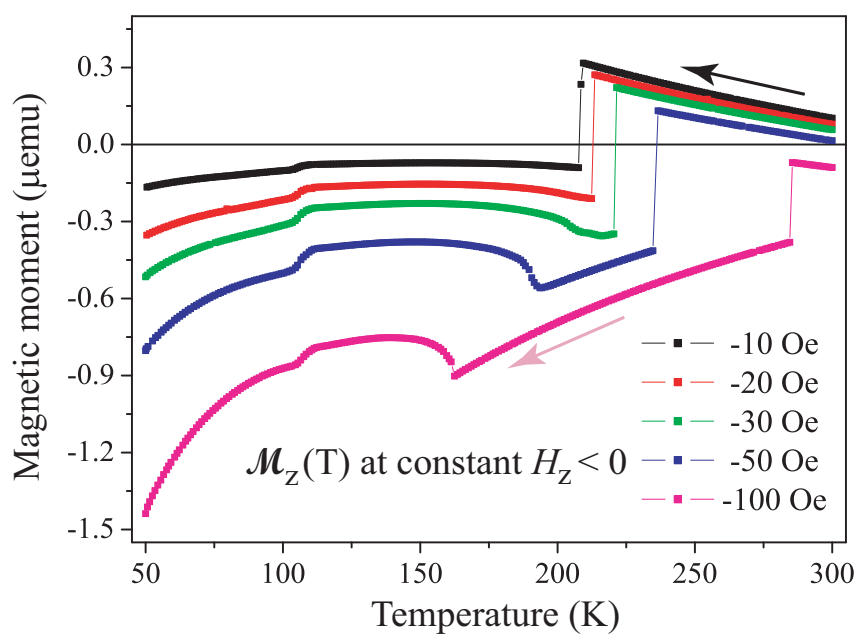

FIG. 5. Out-of-plane $\mathcal{M}_{z}(T)$ at negative values of applied field, $H_{z}<0$. Decreasing temperature scans.
Another qualitative difference between the positive and negative fields is an additional small upward jump that develops on the $\mathcal{M}_{z}(T)$ curves between $T_{2}$ and $T_{1}$ at larger negative fields-see the $H_{z}=-50,-100$ Oe curves in Fig. 5 .

\section{THEORY}

Our measurements reproduce all features that were previously observed in Refs. [42,43] but could not be accounted for by the introduction of the quartic bulk anisotropy term into the energy expression (3). The next goal is to show how they can be consistently explained by the domain structure formation between the $T_{1}$ and $T_{2}$ transitions. We will compare our measurements with the model of Kooy and Enz [27], which assumes a parallel stripe domain structure in the film (see also Ref. [30], Sec. 3.7.3).

First, let us recall how the $\mathcal{M}_{z}\left(H_{z}\right)$ field hysteresis loops in Fig. 4 are explained by the domain structure evolution. When $K_{\text {eff }}(T)$ is positive, the lowest energy state at $H_{z}=0$ is a periodic array of stripe domains [25-32]. Domains with alternating up and down magnetizations lower the dipole-dipole interaction energy. In the absence of an external field, the equilibrium widths of the up and down domains are equal and determined by the material parameters and film thickness. At high temperatures, our films have the smallest magnetization $M(T)$ and largest anisotropy constant $K_{\text {eff }}(T)$. Both factors promote the strong stripe phase, which motivates our use of the Kooy and Enz model [27] that applies to this regime.

If a positive field $H_{z}>0$ is applied to the film, the up domains grow in size, the down domains shrink, and a nonzero net moment $\mathcal{M}_{z}$ is produced. Note that in a theoretical consideration of an infinite film one has to switch from $\mathcal{M}_{z}$ (which is infinite) to the average magnetization $\left\langle M_{z}\right\rangle=\lim _{V \rightarrow \infty}\left(\mathcal{M}_{z} / V\right)$, where $V$ is the film volume. When $H_{z}$ reaches the saturation field $H_{s}$, the width of the up domains diverges, the film assumes a uniformly magnetized state, and $\left\langle M_{z}\right\rangle$ reaches its maximum value of $M(T)$. The value of $H_{s}$ depends on magnetization, magnetic anisotropy energy, and film thickness. A general property of importance for us is the proportionality

$$
H_{s}(T) \sim M(T),
$$

which is a consequence of the fact that the dipole-dipole energy is proportional to $M^{2}(T)$, while Zeeman energy is proportional to the first power of $M(T)$.

In the Kooy and Enz model, the exact field dependence $\left\langle M_{z}\right\rangle\left(H_{z}\right)$ is given by a complicated formula involving a summation of an infinite series. Instead of using it, we will employ an approximate linear expression

$$
\left\langle M_{z}\right\rangle\left(H_{z}, T\right)=\frac{H_{z}}{H_{s}} M(T)
$$

that deviates from the exact formula only for $H_{z}$ close to the saturation field (see Ref. [30], Sec. 3.7.3, Fig. 3.113).

Equation (5) holds when all domain walls can move freely, allowing the magnetic domains to grow and shrink so as to minimize the total energy. However, if one starts an experiment in a saturated state at $H_{z}>H_{s}$ and lowers the field, there is an energy barrier [27,50] preventing the nucleation of domains and making the saturated state metastable below 


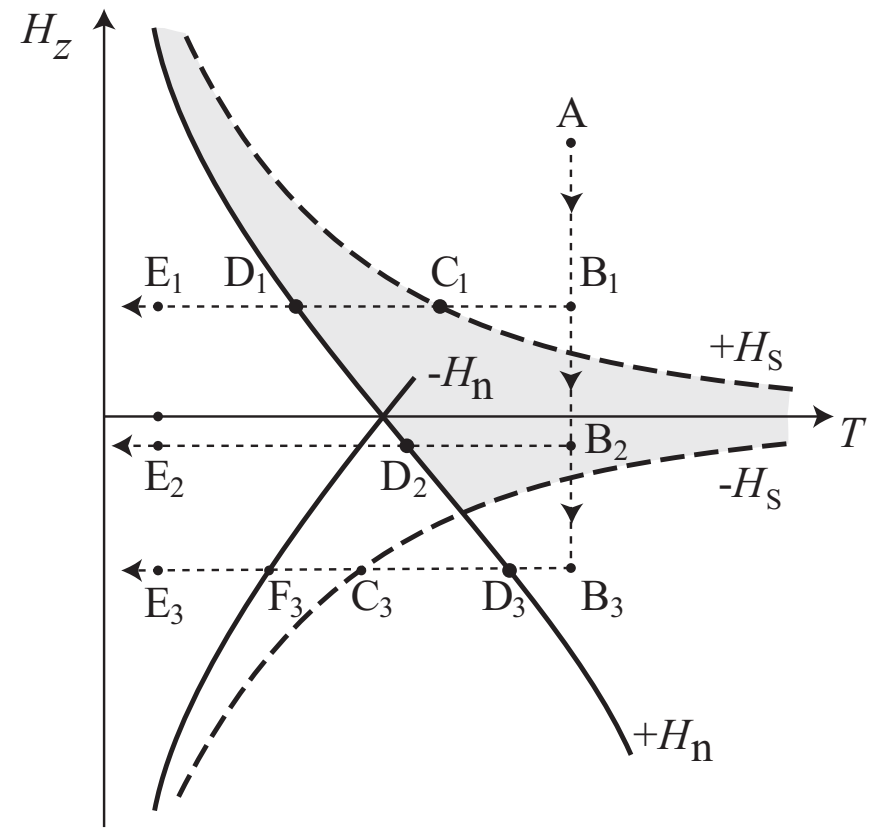

FIG. 6. Qualitative sketch of the theoretical critical fields diagram showing $\pm H_{n}(T)$ (solid line) and $\pm H_{s}(T)$ (dashed lines). Three dotted trajectories $A \rightarrow B_{i} \rightarrow E_{i}(i=1,2,3)$ correspond to the temperature scans $M(T)$ at three different magnitudes of $H_{z}$. If the film is initially saturated in the up direction, temperature hysteresis occurs in the gray region.

$H_{s}$. As a result, the film magnetization remains saturated until $H_{z}$ is lowered to the domain nucleation field $H_{n}$, where it becomes possible for the new domains to appear in the bulk of the film or for the new walls to enter at the film edges. At this point, the domain structure is restored, causing a jump of $\left\langle M_{z}\right\rangle$ from its saturated value $+M(T)$ to a value characterizing the domain structure state in external field. The value of $H_{n}$ is always smaller than that of $H_{s}$. How much smaller depends on the nucleation mechanism. For a given mechanism and variable $M(T)$, the two fields are roughly related by $H_{n}=$ $H_{s}-C / M(T)$ with a mechanism-dependent constant $C$. This is because the magnetic field enters the total energy through a linear in magnetization Zeeman term and has to overcome a specific nucleation barrier. (Particular examples can be found in Refs. [47,48].) The relation shows that $H_{n}$ is positive at large $M$ and negative at small $M$.

The presence of two characteristic fields $H_{s}$ and $H_{n}$ accounts for the shapes of $\left\langle M_{z}\right\rangle\left(H_{z}\right)$ hysteresis loops [47,49]. For $0<H_{n}<H_{s}$, the field hysteresis loops exhibit the "double triangle" shapes [Fig. 4(a)]. For $-H_{s}<H_{n}<0$, the loops have the "triangular tails" shapes [Fig. 4(b)]. In the case of $H_{n}<-H_{s}$, the loops become rectangular.

Since $M(T)$ is known from the experiment, the $H_{s, n}(M)$ dependencies can be converted into $H_{s, n}(T)$. This gives the critical field lines shown in Fig. 6 on the $\left(T, H_{z}\right)$ parameter plane. As the temperature and field are varied according to a certain experimental sequence, the point representing them moves along a trajectory in the $\left(T, H_{z}\right)$ plane. The sequences used in our experiments are represented by the thin dashed trajectories $A \rightarrow B_{i} \rightarrow E_{i}(i=1,2,3)$ in Fig. 6. The common origin of the trajectories, point $A$, corresponds to the initial

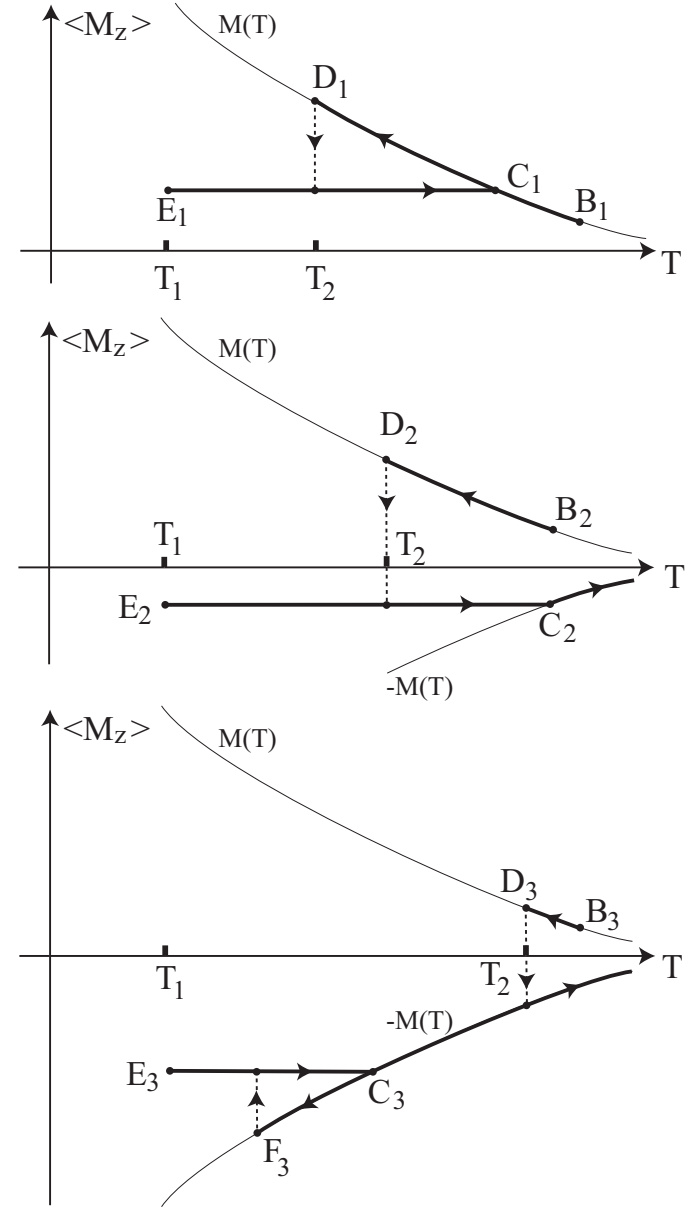

FIG. 7. Theoretical predictions for temperature hysteresis behavior. Points on the curves corresponds to those in Fig. 6. Top panel, sequence $A \rightarrow B_{1} \rightarrow E_{1}$; middle panel, sequence $A \rightarrow B_{2} \rightarrow E_{2}$; bottom panel, sequence $A \rightarrow B_{3} \rightarrow E_{3}$.

saturated state with magnetization pointing up. The field is then dropped to one of the points $B_{i}$ and a temperature scan is performed at $H_{z}=$ const

We now proceed to describe how the existence of the two critical fields $H_{s}$ and $H_{n}$ explains the observed temperature hysteresis.

\section{A. Positive field: $H_{z}>0$}

Theoretical predictions for $\left\langle M_{z}\right\rangle(T)$ are shown in Fig. 7. Consider first the $H_{z}>0$ experiment corresponding to the trajectory $A \rightarrow B_{1} \rightarrow E_{1}$ in Fig. 6. Prediction for $\left\langle M_{z}\right\rangle(T)$ in this case is given in the top panel of Fig. 7. At $B_{1}$ the inequality $H_{z}>H_{n}$ holds, so the domains are not yet nucleated and the film stays in a saturated state with magnetization pointing up. As the temperature is decreased at constant $H_{z}$, the uniform state remains stable with respect to the domain structure formation until the $H_{n}$ line is crossed at the point $D_{1}$. On the $B_{1} \rightarrow D_{1}$ interval, $\left\langle M_{z}\right\rangle(T)$ is equal to the full $+M(T)$ value and thus grows with decreasing temperature.

At $D_{1}$, the sample develops a domain structure, so $\left\langle M_{z}\right\rangle$ drops from $+M\left(T_{D 1}\right)$ to the equilibrium domain structure value $\left\langle M_{z}\right\rangle\left(T_{D 1}\right)$ given by Eq. (5). This produces a downward jump on the graph. 
Between $D_{1}$ and $E_{1}$, the film stays in the stripe domain state. Here $\left\langle M_{z}\right\rangle$ is given by (5) with a fixed $H_{z}$ and temperature-dependent $H_{s}(T)$ and $M(T)$. Because of the property (4), the theory predicts $\left\langle M_{z}\right\rangle(T)=$ const on the $D_{1} \rightarrow E_{1}$ interval.

When an increasing temperature scan is performed along the $E_{1} \rightarrow D_{1} \rightarrow C_{1} \rightarrow B_{1}$ trajectory, the same sequence of events goes in the reverse order, except that at $D_{1}$ the film is already in the lowest energy domain state and has no reason to switch to a saturated state. The value of $\left\langle M_{z}\right\rangle$ does not jump at $D_{1}$ but remains constant. When the trajectory of the system in the $\left(T, H_{z}\right)$ plane reaches the $H_{s}$ line at $C_{1}$, the width of up domains diverges and a saturated state of the film is established. At this point, the upper and the lower branches of the triangular hysteresis loop join each other.

\section{B. Negative field: $\boldsymbol{H}_{z}<0$}

For the case of $H_{z}<0$, we will consider two typical trajectories: $A \rightarrow B_{2} \rightarrow E_{2}$ and $A \rightarrow B_{3} \rightarrow E_{3}$ in Fig. 6. They cross different numbers of critical lines, and our theory predicts qualitatively different $\left\langle M_{z}\right\rangle(T)$ dependencies for them.

For the $A \rightarrow B_{2} \rightarrow E_{2}$ trajectory, the predicted $\left\langle M_{z}\right\rangle(T)$ is shown in the middle panel of Fig. 7. Although a negative $H_{z}$ is applied, the domains cannot form at $B_{2}$ since $H_{n}\left(B_{2}\right)<H_{z}<$ 0 . The film is in the state of uniform up magnetization. As the downward temperature sweep begins, the magnetization of the film stays uniform with $\left\langle M_{z}\right\rangle=+M(T)$ until the trajectory crosses the $H_{n}$ line at $D_{2}$, where a jump into the domain structure state occurs. In that state, the average magnetization given by Eq. (5) is negative. It stays constant upon further temperature decrease according to formulas (4) and (5). If an increasing temperature scan $E_{2} \rightarrow D_{2} \rightarrow B_{2}$ is performed, no change is expected at $D_{2}$ since the film is already in the lowest energy domain state. The magnetization stays negative and constant.

The situation is more complex on the $A \rightarrow B_{3} \rightarrow E_{3}$ trajectory, for which the predicted $\left\langle M_{z}\right\rangle(T)$ is shown in the bottom panel of Fig. 7. Similar to the case above, at $B_{3}$ the film is in a uniformly magnetized state with up magnetization. At the point $D_{3}$, domain formation becomes possible but, in contrast with the situation at $D_{2}$, here $H_{z}<-H_{s}$, so now the widths of the down domains diverge and the uniform downmagnetized state of the film is established: A full reversal of the magnetization from $+M\left(T_{D 3}\right)$ to $-M\left(T_{D 3}\right)$ takes place via the domain wall motion mechanism [50]. As the temperature is lowered further, the film remains saturated in the down direction, and $\left\langle M_{z}\right\rangle=-M(T)$ gets progressively more negative. For the down-magnetized film, the relevant nucleation field is $-H_{n}(T)$, so the magnetization remains uniform on the $D_{3} \rightarrow F_{3}$ interval. At $F_{3}$, the film transitions into a stripe domain state with the average magnetization given by Eq. (5). This produces an upward jump of $\left\langle M_{z}\right\rangle$. After this jump, the magnetization remains temperature-independent in the interval $F_{3} \rightarrow E_{3}$ according to the formulas (4) and (5).

\section{DISCUSSION}

We now compare the experimental data and the theory predictions for $\left\langle M_{z}\right\rangle(T)$ and argue that the experiment is well explained by the theory, apart from some extrinsic factors and minor inaccuracies of the approximations used in the analysis.

Experimental curves in Fig. $3\left(H_{z}>0\right)$ should be compared with the theoretical curve in the top panel of Fig. 7. Experimental curves in Fig. 5 measured at $H_{z}=-10,-20,-30$ Oe should be compared with the theoretical curve in the middle panel of Fig. 7. Finally, experimental curves for $H_{z}=$ $-50,-100$ Oe in Fig. 5 should be compared with the theoretical curve in the bottom panel of Fig. 7. We see that in all cases the shapes of the $\left\langle M_{z}\right\rangle(T)$ loops in the temperature range above $T_{1}$ are well reproduced by the theory, apart from a single apparent discrepancy: the progressive parallel shifts of the experimental curves upward for $H_{z}>0$ and downward for $H_{z}<0$. This discrepancy will be discussed later in this section.

Importantly, in all experiments the film exhibits approximately temperature-independent value of $\left\langle M_{z}\right\rangle$ in the temperature regions where our theory identifies its state as multidomain. In the regions where the state of the film is identified as uniformly magnetized, $\left\langle M_{z}\right\rangle(T)= \pm M(T)+$ $f\left(H_{z}\right)$, where $f$ is the parallel shift mentioned above. Apart from the shift, both properties are in accord with our theory.

The domain structure picture is also consistent with the observation of a large difference between the field derivatives $d T_{1} / d H_{\|}$and $d T_{2} / d H_{z}$. In a theory based on the energy function (3), those derivatives would be of the same order, but in our approach, the value of $d T_{2} / d H_{z}$ comes from the $H_{n}(M(T))$ dependence, i.e., from the physics of domain wall nucleation, and does not have to be related to $d T_{1} / d H_{\|}$, which is determined by the bulk parameters of the film.

Let us now return to the vertical parallel shift $f\left(H_{z}\right)$. We attribute it to the paramagnetic contribution of the substrate, which is indeed positive for $H_{z}>0$ and negative for $H_{z}<0$. At $H_{z}=-100 \mathrm{Oe}$, this effect is so strong that the whole $\left\langle M_{z}\right\rangle(T)$ curve in Fig. 5 is shifted into the region of negative average magnetization. However, apart from this overall shift, its shape is in accord with the theoretical prediction shown in the bottom panel of Fig. 7.

A less visible discrepancy between theory and experiment manifests itself in the deviation of the lower branch of the triangular hysteresis loop $\left\langle M_{z}\right\rangle(T)$ in Fig. 3 from a temperature-independent constant predicted in the top panel of Fig. 7. This is a deficiency of our theoretical treatment caused by the approximation (5). Exact $\left\langle M_{z}\right\rangle\left(H_{z}\right)$ dependence becomes nonlinear whenever $H_{z}$ is close to $H_{s}[27,30]$, i.e., when the system is close to the point $C_{1}$ in the top panel of Fig. 7. This is exactly where the largest discrepancy is observed. We assume that the use of exact $\left\langle M_{z}\right\rangle\left(H_{z}\right)$ expression would have reproduced the experimental results better.

As the downward temperature sweep is continued, the system eventually approaches the $T_{1}$ transition. Surprisingly, experimental $\left\langle M_{z}\right\rangle(T)$ curves exhibit a signature at $T_{1}$ : a small jump and in the case of $H_{z} \neq 0$ an increase of $\left\langle M_{z}\right\rangle$ upon further cooling. Are those features explained by the theory? Consider the $H_{z}=0$ measurement. Here $\left\langle M_{z}\right\rangle=0$ must hold both above $T_{1}$ in a multidomain state with equal sizes of up and down domains, and below $T_{1}$, where magnetization is directed in plane. The $T_{1}$ transition should thus be invisible on 
an $\left\langle M_{z}\right\rangle(T)$ curve. The actual presence of its signature can be nevertheless explained by a small misalignment of the film. When the $z$ axis of the film is slightly tilted with respect to the measurement axis $z^{\prime}$ of the magnetometer, a purely in-plane magnetization produces a small component along $z^{\prime}$, recorded as of $M_{z}$. In the present study, we do not concentrate on the properties of the $T_{1}$ transition, but from Fig. 2 one sees that it has a first-order character. Therefore, a jump of $M_{\|}$at the $T_{1}$ transition produces a small parasitic jump of $M_{z}$ seen in experiments. For $H_{z} \neq 0$, one would need to know the type of domain structure above $T_{1}$ and have a theory of its response to $H_{z}$ to explain the $\left\langle M_{z}\right\rangle(T)$ dependence and the shift of transition point produced by the applied field. The model of strong stripe phase [27] that worked well for us near $T_{2}$ is not guaranteed to apply right above $T_{1}$ : As mentioned in the introduction, the theory of the $T_{1}$ transition that happens close to the $K_{\text {eff }}=0$ point may be nontrivial [37-41]. At this time, there is not enough experimental information to suggest such theory with certainty, and we leave this task for the future research.

One may also ask why the stipulated misalignment of the film plane does not produce a signature of the $T_{2}$ transition on the $M_{\|}\left(H_{\|}\right)$curves in Fig. 2. Our explanation is as follows. As long as the misalignment is small enough, the parasitic perpendicular component $H_{z}$ satisfies $\left|H_{z}\right|<H_{s}(T)$ for all temperatures. As a result, the film never switches from a stripe domain state to a uniform saturated state. Accordingly, the jump at $T_{2}$ never occurs, as it can only happen from a saturated state to the multidomain state but not vice versa. In other words, $M_{z}(T)$ stays on the lower branch of the triangular temperature hysteresis loop and thus changes continuously during the temperature sweep.

\section{CONCLUSIONS}

We have shown how the previously unexplained features of the double orientation transition in thin garnet films can be understood in terms of the stripe-domain structure formation and evolution. In our theoretical picture, both the lowand the high-temperature transitions occur between a uniform state and a multidomain state. The essential difference between them is that the high-temperature transition reflects the physics of the domain nucleation, while the low-temperature transition is governed by the bulk properties.

When garnet films are used as elements of heavy metal/ferromagnetic insulator bilayer devices, the stripedomain state will influence the heavy metal conductivity tensor and should allow one to study the contribution of domain walls to the interplay of electric and magnetic properties in the device, possibly leading to novel types of tunability by the applied magnetic field.

\section{ACKNOWLEDGMENTS}

The authors are grateful to P. E. Wigen for stimulating their interest in the subject. G. N. Kakazei acknowledges the Network of Extreme Conditions Laboratories (NECL) and Portuguese Foundation of Science and Technology (FCT) support through the Projects No. NORTE-01-0145FEDER-022096 and No. PTDC/FIS-MAC/31302/2017. Ya. B. Bazaliy was supported by NSF Grant No. DMR-0847159.
[1] Y. Kajiwara, K. Harii, S. Takahashi, J. Ohe, K. Uchida, M. Mizuguchi, H. Umezawa, H. Kawai, K. Ando, K. Takanashi, S. Maekawa, and E. Saitoh, Transmission of electrical signals by spin-wave interconversion in a magnetic insulator, Nature (London) 464, 262 (2010).

[2] K. Uchida, H. Adachi, Y. Kajiwara, S. Maekawa, and E. Saitoh, Spin-wave spin current in magnetic insulators, in Recent Advances in Magnetic Insulators-from Spintronics to Microwave Applications, edited by M. Wu and A. Hoffmann, Solid State Physics Vol. 64 (Academic Press, 2013).

[3] A. V. Chumak, V. I. Vasyuchka, A. A. Serga, and B. Hillebrands, Magnon spintronics, Nat. Phys. 11, 453 (2015).

[4] V. E. Demidov, S. Urazhdin, G. de Loubens, O. Klein, V. Cros, A. Anane, and S. O. Demokritov, Magnetization oscillations and waves driven by pure spin currents, Phys. Rep. 673, 1 (2017).

[5] M. Althammer, Pure spin currents in magnetically ordered insulator/normal metal heterostructures, J. Phys. D: Appl. Phys. 51, 313001 (2018).

[6] A. Fert and F. N. Van Dau, Spintronics, from giant magnetoresistance to magnetic skyrmions and topological insulators, C. R. Phys. 20, 817 (2019).

[7] A. Hirohata, K. Yamada, Y. Nakatani, I.-L. Prejbeanu, B. Diény, P. Pirroe, and B. Hillebrands, Review on spintronics: Principles and device applications, J. Magn. Magn. Mater. 509, 166711 (2020).
[8] C. Tang, P. Sellappan, Y. Liu, Y. Xu, J. E. Garay, and Jing Shi, Anomalous Hall hysteresis in $\mathrm{Tm}_{3} \mathrm{Fe}_{5} \mathrm{O}_{12} / \mathrm{Pt}$ with straininduced perpendicular magnetic anisotropy, Phys. Rev. B 94, 140403(R) (2016).

[9] Y. K. Liu, H. F. Wong, K. K. Lam, K. H. Chan, C. L. Mak, and $\mathrm{C}$. W. Leung, Anomalous Hall effect in $\mathrm{Pt} / \mathrm{Tb}_{3} \mathrm{Fe}_{5} \mathrm{O}_{12}$ heterostructure: Effect of compensation point, J. Magn. Magn. Mater. 458, 235 (2018).

[10] V. H. Ortiz, M. Aldosary, J. Li, Y. Xu, M. I. Lohmann, P. Sellappan, Y. Kodera, J. E. Garay, and J. Shi, Systematic control of strain-induced perpendicular magnetic anisotropy in epitaxial europium and terbium iron garnet thin films, APL Mater. 6, 121113 (2018).

[11] C. O. Avci, A. Quindeau, M. Mann, C.-F. Pai, C. A. Ross, and G. S. D. Beach, Spin transport in as-grown and annealed thulium iron garnet/platinum bilayers with perpendicular magnetic anisotropy, Phys. Rev. B 95, 115428 (2017).

[12] C. O. Avci, A. Quindeau, C.-F. Pai, M. Mann, L. Caretta, A. S. Tang, M. C. Onbasli, C. A. Ross, and G. S. D. Beach, Currentinduced switching in a magnetic insulator, Nat. Mater. 16, 309 (2017).

[13] C. O. Avci, E. Rosenberg, M. Baumgartner, L. Beran, A. Quindeau, P. Gambardella, C. A. Ross, and G. S. D. Beach, Fast switching and signature of efficient domain wall motion driven by spin-orbit torques in a perpendicular anisotropy magnetic insulator/Pt bilayer, Appl. Phys. Lett. 111, 072406 (2017). 
[14] Q. Shao, C. Tang, G. Yu, A. Navabi, H. Wu, C. He, J. Li, P. Upadhyaya, P. Zhang, S. A. Razavi, Q. L. He, Y. Liu, P. Yang, S. K. Kim, C. Zheng, Y. Liu, L. Pan, R. K. Lake, X. Han, Y. Tserkovnyak, J. Shi, and K. L. Wang, Role of dimensional crossover on spin-orbit torque efficiency in magnetic insulator thin films, Nat. Commun. 9, 3612 (2018).

[15] N. Kumar, N. G. Kim, Y. A. Park, N. Hur, J. H. Jung, K. J. Han, and K. J. Yee, Epitaxial growth of terbium iron garnet thin films with out-of-plane axis of magnetization, Thin Solid Films 516, 7753 (2008).

[16] M. Kubota, A. Tsukazaki, F. Kagawa, K. Shibuya, Y. Tokunaga, M. Kawasaki, and Y. Tokura, Stress-induced perpendicular magnetization in epitaxial iron garnet thin films, Appl. Phys. Express 5, 103002 (2012).

[17] H. Asada, A. Kuwahara, N. Sakata, T. Ono, T. Ishibashi, A. Meguro, T. Hashinaka, K. Kishimoto, and T. Koyanagi, Longitudinal spin Seebeck effect in $\mathrm{Nd}_{2} \mathrm{BiFe}_{5-x} \mathrm{Ga}_{x} \mathrm{O}_{12}$ prepared on gadolinium gallium garnet (001) by metal organic decomposition method, J. Appl. Phys. 117, 17C724 (2015).

[18] A. Quindeau, C. O. Avci, W. Liu, C. Sun, M. Mann, A. T. Tang, M. C. Onbasli, D. Bono, P. M. Voyles, G. S. D. Beach, Y. $\mathrm{Xu}$, and C. A. Ross, $\mathrm{Tm}_{3} \mathrm{Fe}_{5} \mathrm{O}_{12} / \mathrm{Pt}$ heterostructures with perpendicular magnetic anisotropy for spintronic applications, Adv. Elect. Mater. 3, 1600376 (2017).

[19] C. N. Wu, C. C. Tseng, K. Y. Lin, C. K. Cheng, S. L. Yeh, Y. T. Fanchiang, M. Hong, and J. Kwo, High-quality singlecrystal thulium iron garnet films with perpendicular magnetic anisotropy by off-axis sputtering, AIP Adv. 8, 055904 (2018).

[20] Y. Rao, D. Zhang, H. Zhang, L. Jin, Q. Yang, Z. Zhong, M. Li, C. Hong, and B. Ma, Thickness dependence of magnetic properties in submicron yttrium iron garnet films, J. Phys. D: Appl. Phys. 51, 435001 (2018).

[21] E. R. Rosenberg, L. Beran, C. O. Avci, C. Zeledon, B. Song, C. Gonzalez-Fuentes, J. Mendil, P. Gambardella, M. Veis, C. Garcia, G. S. D. Beach, and C. A. Ross, Magnetism and spin transport in rare-earth-rich epitaxial terbium and europium iron garnet films, Phys. Rev. Mater. 2, 094405 (2018).

[22] S. M. Elhamali, N. B. Ibrahim, and S. Radiman, Structural, optical, and magnetic properties of YIG and TbErIG nanofilms prepared using a sol-gel method, Mater. Res. Bull. 112, 66 (2019).

[23] N. I. Mezin, Y. I. Nepochatykh, N. Y. Starostyuk, and S. V. Yampolskii, Magnetic susceptibility anomalies and inverse magnetic transitions in iron-enriched garnet films, J. Magn. Magn. Mater. 476, 447 (2019).

[24] Q. Shao, Y. Liu, G. Yu, S. K. Kim, X. Che, C. Tang, Q. L. He, Y. Tserkovnyak, J. Shi, and K. L. Wang, Topological Hall effect at above room temperature in heterostructures composed of a magnetic insulator and a heavy metal, Nat. Elect. 2, 182 (2019).

[25] C. Kittel, Theory of the structure of ferromagnetic domains in films and small particles, Phys. Rev. 70, 965 (1946).

[26] Z. Málek and V. Kamberský, On the theory of the domain structure of thin films of magnetically uni-axial materials, Czech. J. Phys. 8, 416 (1958).

[27] C. Kooy and U. Enz, Experimental and theoretical study of the domain conifguration in thin layers of $\mathrm{BaFe}_{12} \mathrm{O}_{19}$, Philips Res. Rep. 15, 7 (1960).

[28] M. W. Muller, Distribution of magnetization in a ferromagnet, Phys. Rev. 122, 1485 (1961).
[29] V. Gehanno, Y. Samson, A. Marty, B. Gilles, and A. Chamberod, Magnetic susceptibility and magnetic domain configuration as a function of the layer thickness in epitaxial $\mathrm{FePd}(001)$ thin films ordered in the L1(0) structure, J. Magn. Magn. Mater. 172, 26 (1997).

[30] A. Hubert and R. Schäfer, Magnetic Domains: The Analysis of Magnetic Microstrctures (Springer, Berlin, 1998).

[31] M. Labrune and A. Thiaville, Micromagnetic structure in multilayer films with moderate perpendicular anisotropy, Eur. Phys. J. B 23, 17 (2001).

[32] D. Clarke, Thickness dependence of the reorientation phase transition, arXiv:0705.2600 [cond-mat.mtrl-sci].

[33] R. Allenspach, Ultrathin films: Magnetism on the microscopic scale, J. Magn. Magn. Mater. 129, 160 (1994).

[34] D. Pescia and V. L. Pokrovsky, Perpendicular Versus In-Plane Magnetization in a 2D Heisenberg Monolayer at Finite Temperatures, Phys. Rev. Lett. 65, 2599 (1990); P. Politi, A. Rettori, and M. G. Pini, Comment, ibid. 70, 1183 (1993); A. P. Levanyuk and N. Garcia, Comment, ibid. 70, 1184 (1993); D. Pescia and V. L. Pokrovsky, Reply, ibid. 70, 1185 (1993).

[35] Y. Yafet and E. M. Gyorgy, Ferromagnetic strip domains in an atomic monolayer, Phys. Rev. B 38, 9145 (1988).

[36] A. Kashuba and V. L. Pokrovsky, Stripe Domain Structures in a Thin Ferromagnetic Film, Phys. Rev. Lett. 70, 3155 (1992); Stripe domain structures in a thin ferromagnetic film, Phys. Rev. B 48, 10335 (1993).

[37] D. Clarke, O. A. Tretiakov, and O. Tchernyshyov, Stripes in thin ferromagnetic films with out-of-plane anisotropy, Phys. Rev. B 75, 174433 (2007).

[38] P. J. Jensen and K. H. Bennemann, Direction of the magnetization of thin films and sandwiches as a function of temperature, Phys. Rev. B 42, 849 (1990).

[39] P. J. Jensen and K. H. Bennemann, On direction of the magnetization of thin films and sandwiches as a function of temperature-II, Solid State Comm. 83, 1057 (1992).

[40] A. Moschel and K. D. Usadel, Influence of the dipole interaction on the direction of the magnetization in thin ferromagnetic-films, Phys. Rev. B 49, 12868 (1994).

[41] A. Moschel and K. D. Usadel, Reorientation transitions of first order and second order in thin ferromagnetic films, Phys. Rev. B 51, 16111 (1995).

[42] R. E. Bornfreund, D. C. Khan, P. E. Wigen, M. PardaviHorvath, J. Ings, and R. Belt, Spin reorientation in $\mathrm{Tm}_{2.14} \mathrm{Bi}_{0.80} \mathrm{~Pb}_{0.06} \mathrm{Fe}_{3.1} \mathrm{Ga}_{1.9} \mathrm{O}_{12}$ thin films, IEEE Trans. Magn. 28, 2991 (1992).

[43] R. E. Bornfreund, D. C. Khan, P. E. Wigen, M. Pardavi-Horvath, J. Ings, and R. Belt, Effects of a magnetic field on spin reorientation in BiGa-TmIG films, J. Magn. Magn. Mater. 151, 181 (1995).

[44] H. Horner and C. Varma, Nature of Spin-Reorientation Transitions, Phys. Rev. Lett. 20, 845 (1968).

[45] K. P. Belov, A. K. Zvezdin, A. M. Kadomtseva, and R. V. Levitin, Orientation Phase Transitions in Rare Earth Magnetic Materials (Nauka, Moscow, 1979).

[46] L. T. Tsymbal,Y. B. Bazaliy, G. N. Kakazei, F. J. Palomares, and P. E. Wigen, Magnetic hysteresis in $\mathrm{ErFeO}_{3}$ near the low temperature erbium ordering transition, IEEE Trans. Magn. 44, 2933 (2008). 
[47] L. T. Tsymbal, G. N. Kakazei, and Ya. B. Bazaliy, Singledomain-wall states in millimeter-scale samples of $\mathrm{ErFeO}_{3}$, Phys. Rev. B 79, 092414 (2009).

[48] Y. B. Bazaliy and L. T. Tsymbal, Triangular hysteresis loops in the spin-rotation region of orthoferrites, Low Temp. Phys. 36, 798 (2010).
[49] L. T. Tsymbal, Y. B. Bazaliy, G. N. Kakazei, and S. V. Vasiliev, Mechanisms of magnetic and temperature hysteresis in $\mathrm{ErFeO}_{3}$ and $\mathrm{TmFeO}_{3}$ single crystals, J. Appl. Phys. 108, 083906 (2010).

[50] E. Kondorsky, On hysteresis in ferromagnetics, J. Phys. USSR 2, 161 (1940). 